\title{
Assessment of acute, 14-day, and 13-week repeated oral dose toxicity of Tiglium seed extract in rats
}

Jun-Won Yun ${ }^{1 \dagger}$, Euna Kwonn ${ }^{2 \dagger}$, Yun-Soon Kim², Seung-Hyun Kim², Ji-Ran You ${ }^{2}$, Hyoung-Chin Kim³ ${ }^{3}$ Jin-Sung Park², Jeong-Hwan Che ${ }^{4}$, Sang-Koo Lee ${ }^{2}$, Ja-June Jang ${ }^{5}$, Hyeon Hoe Kim ${ }^{6}$ and Byeong-Cheol Kang 2,4,7,8,9*

\begin{abstract}
Background: Seed of mature Croton tiglium Linne, also known as Tiglium seed (TS), has been widely used as a natural product due to its several health beneficial properties including anti-tumor and antifungal activities. Despite its ethnomedicinal beneficial properties, toxicological information regarding TS extract, especially its long-term toxicity, is currently limited. Therefore, the objective of the present study was to evaluate acute and subchronic toxicity of TS extract in rats after oral administration following test guidelines of the Organization for Economic Cooperation and Development (OECD).

Methods: Toxicological properties of TS extract were evaluated by toxicity assays to determine its single-dose acute toxicity $(125,250,500,1000$, or 2000 mg/kg), 14-day repeated-dose toxicity $(125,250,500,1000$, or $2000 \mathrm{mg} / \mathrm{kg})$ and 13-week repeated-dose toxicity $(31.25,62.5,125,250$, and $500 \mathrm{mg} / \mathrm{kg})$ in Sprague-Dawley rats and F344 rats. Hematological, serum biochemical, and histopathological parameters were analyzed to determine its median lethal dose $\left(\mathrm{LD}_{50}\right)$ and no-observed-adverse-effect-level (NOAEL).

Results: Oral single dose up to $2000 \mathrm{mg} / \mathrm{kg}$ of TS extract resulted in no mortalities or abnormal clinical signs. In 13-week toxicity study, TS extract exhibited no dose-related changes (mortality, body weight, food/water consumption, hematology, clinical biochemistry, organ weight, or histopathology) at dose up to $500 \mathrm{mg} / \mathrm{kg}$, the highest dosage level suggested based on 14-day repeat-dose oral toxicity study.

Conclusion: Acute oral $\mathrm{LD}_{50}$ of TS extract in rats was estimated to be greater than $2000 \mathrm{mg} / \mathrm{kg}$. NOAEL of TS extract administered orally was determined to be $500 \mathrm{mg} / \mathrm{kg} /$ day in both male and female rats. Results from these acute and subchronic toxicity assessments of TS extract under Good Laboratory Practice regulations indicate that TS extract appears to be safe for human consumption.
\end{abstract}

Keywords: Tiglium seed, Acute, Subchronic, Toxicity

\footnotetext{
* Correspondence: bckang@snu.ac.kr

† Jun-Won Yun and Euna Kwon contributed equally to this work.

2Department of Experimental Animal Research, Biomedical Research Institute,

Seoul National University Hospital, 101 Daehak-ro, Jongno-gu, Seoul 03080,

Republic of Korea

${ }^{4}$ Biomedical Center for Animal Resource and Development, Seoul National

University College of Medicine, 101 Daehak-ro, Jongno-gu, Seoul 03080

Republic of Korea

Full list of author information is available at the end of the article
}

(c) The Author(s). 2018 Open Access This article is distributed under the terms of the Creative Commons Attribution 4.0 International License (http://creativecommons.org/licenses/by/4.0/), which permits unrestricted use, distribution, and reproduction in any medium, provided you give appropriate credit to the original author(s) and the source, provide a link to the Creative Commons license, and indicate if changes were made. The Creative Commons Public Domain Dedication waiver (http://creativecommons.org/publicdomain/zero/1.0/) applies to the data made available in this article, unless otherwise stated. 


\section{Background}

Croton, a large genus belonging to family Euphorbiaceae, is widespread in tropical regions of Southeast Asia and China. Many previous studies have reported beneficial pharmacological activities of Croton species for treating diabetes, gastritis, and digestive disorders [1-5]. Sandoval et al. [6] have demonstrated that Croton tiglium extract possesses growth inhibitory or antiproliferative effects on cancer. Ethanolic extract of Croton tiglium is also known to exhibit antifungal activities for treating dermatophytes caused by Trichophyton mentagrophytes, T. rubrum, and Epidermophyton floccosum [7]. Croton tiglium Linne has been recorded as a traditional Chinese medicine for gastrointestinal disorders, rheumatism, headache, peptic ulcer, and visceral pain in the second century B.C. [8-11]. Tiglium seed (TS), a seed of mature Croton tiglium Linne, is known to contain croton oil extensively used as laxative or purgative $[12,13]$.

Plant-derived natural products are attracting significant attention around the world due to the perception that they are safe 'natural' drugs for promoting health without showing side effects. In addition, it is easy to obtain them from many grocery stores without having a prescription [14-20]. However, it has been demonstrated that many traditional beneficial herbs have genotoxic or systemic (liver and kidney) toxicity [21-25]. Thus, it is necessary to conduct comprehensive safety analysis under strict guidelines for herbal medicine available on the market. Although many people believe that oriental herbs are safe, data on systemic oral toxicity of TS extract as a natural therapeutic product with a variety of beneficial properties are lacking. Therefore, the objective of this study was to determine the median lethal dose $\left(\mathrm{LD}_{50}\right)$ and no-observed-adverse-effect-level (NOAEL) of TS extract after oral administration in Sprague-Dawley (SD) rats and F344 rats by conducting single-dose acute toxicity and 13-week repeated-dose toxicity studies following Good Laboratory Practice (GLP) regulations [26] and Organization for Economic Cooperation and Development (OECD) test guideline [27].

\section{Methods}

\section{Test substance and animals}

TS collected from China were purchased at a Kyung-dong traditional herbal market (Seoul, Korea) and authenticated by Professor Chang Soo Yook of Kyung Hee University, an expert in the field of herbal medicine. Voucher specimen (SNUH-GLP 04014) has been deposited at the Department of Experimental Animal Research, Biomedical Research Institute, Seoul National University Hospital in Korea. Stepwise extraction of TS was performed using solvents of increasing polarity (hexane and ethyl acetate) [28]. Briefly, TS was ground into powder after peeling off testa. After removing oil from TS, hexane was added to TS and kept at room temperature for $24 \mathrm{~h}$. They were then filtered through filter paper and dried in clean and dark area at room temperature. After adding ethyl acetate, extracts were stored for $24 \mathrm{~h}$, filtered, and dried. Distilled water (DW) was added to the dried extract and stirred at $4{ }^{\circ} \mathrm{C}$ for $72 \mathrm{~h}$. They were filtered with membrane paper (0.24 $\mu \mathrm{m}$, Eyela Tokyo Rikakikai Co., Ltd., Tokyo, Japan) and concentrated by evaporation to half volume. Finally, the TS extract was frozen dried for $24 \mathrm{~h}$ and dissolved in $1 \%$ methylcellulose $(\mathrm{MC})$ before oral administration.

Specific pathogen-free Sprague-Dawley (SD) rats and F344 rats were obtained from Orient Bio (Seongnam, Korea) and SLC (Hamamatsu, Japan), respectively. The animals were housed under controlled conditions (temperature, $22 \pm 2{ }^{\circ} \mathrm{C}$; humidity, 40-60\%) in the experimental animal facility at Seoul National University Hospital accredited by AAALAC International (\#001169) in accordance with Guide for the Care and Use of Laboratory Animals, 8th edition [29]. These animals were allowed free access to their diet (LabDiet 5002 Certified Rodent Diet, PMI Nutrition International, St. Louis, MO, USA) and tap water with a $12 \mathrm{~h}$ light:dark cycle. The rats were adapted to this environment for 1 week prior to study initiation. The animal studies were approved by Institutional Animal Care and Use Committee of the Biomedical Research Institute at Seoul National University Hospital and conducted under the guideline published by OECD [27] as well as GLP regulations for Nonclinical Laboratory Studies of Korea Food Drug Administration [26].

\section{Experimental design for single oral dose toxicity study}

For single oral dose toxicity study, healthy male and female SD rats (7-week old) were randomly assigned to six groups (3/sex/group). Vehicle (1\% MC) or graded doses of TS extract $(125,250,500,1000$, and $2000 \mathrm{mg} / \mathrm{kg}$ of body weight) were administered to rats by oral gavage once at dose of $10 \mathrm{ml} / \mathrm{kg}$ of body weight. The rats were observed for mortality and clinical signs every hour for $6 \mathrm{~h}$ after dosing during the first $24 \mathrm{~h}$ and then once daily for a total of 14 days. Body weights were recorded on days $0,1,7$, and 14 after the treatment. At study termination, all rats were euthanized by isoflurane (2\% to $5 \%)$ inhalation and their organs were then collected for macroscopic necropsy examination.

\section{Experimental design for repeated oral dose toxicity study} For 14-day repeat-dose toxicity study, healthy male and female F344 rats (7-week old) were randomly assigned to six groups (5/sex/group). Vehicle (1\% MC) or graded doses of TS extract (125, 250, 500, 1000, and $2000 \mathrm{mg} /$ $\mathrm{kg}$ of body weight) were administered to rats by oral gavage once daily for 14 days at dose of $10 \mathrm{ml} / \mathrm{kg}$ of body weight. The rats were observed daily for mortality 
and clinical signs for 14 days. Body weights were recorded on days 0,7 , and 14 after the treatment. At study termination, all rats were euthanized by isoflurane $(2 \%$ to $5 \%$ ) inhalation and their organs were then collected for macroscopic necropsy examination. Organ weights were measured for the following: liver, kidney, testis, thymus, heart, and lung.

For 13-week repeat-dose toxicity study in accordance with OECD guideline 408 [27], healthy male and female F344 rats (7-week old) were randomly assigned to six groups (10/sex/group). Vehicle (1\% MC) or graded doses of TS extract $(31.25,62.5,125,250$, and $500 \mathrm{mg} / \mathrm{kg}$ of body weight) were administered to rats by oral gavage once daily for 13 weeks at dose of $10 \mathrm{ml} / \mathrm{kg}$ of body weight. The rats were observed daily for clinical signs including mortality, general appearance, and behavioral abnormality until terminal sacrifice. Body weights and food/water consumption were recorded weekly throughout the study. At study termination, all rats were euthanized by isoflurane ( $2 \%$ to $5 \%$ ) inhalation for blood sample collection.

\section{Hematology and serum biochemistry}

After blood samples were collected into EDTA tubes, the following hematological parameters were analyzed using a Vet $\mathrm{abc}^{\mathrm{Ts}}$ Animal Blood Counter (Horiba ABX, Montpellier, France): white blood cell (WBC), red blood cell (RBC), hemoglobin (HGB), hematocrit (HCT), platelet (PLT), mean corpuscular volume (MCV), mean corpuscular hemoglobin $(\mathrm{MCH})$, mean corpuscular hemoglobin concentration $(\mathrm{MCHC})$, and differential WBC (neutrophils, eosinophils, basophils, lymphocytes, and monocytes). The following serum biochemistry parameters were also measured using serum immediately separated from whole blood samples collected into tubes without anticoagulant with an automatic chemistry analyzer (Hitachi 7070, Hitachi, Tokyo, Japan): blood urea nitrogen (BUN), total cholesterol (TC), total bilirubin (TB), total protein (TP), albumin, alkaline phosphatase (ALP), aspartate transaminase (AST), alanine transaminase (ALT), creatinine, and triglyceride (TG).

\section{Gross findings, organ weights, and histopathological assessments}

At necropsy, the animals were sacrificed to analyze the gross and microscopic features of the internal organs. The liver, kidney, testis, thymus, heart, and lung were excised and weighed. Relative organ weight to terminal body weight was also calculated. Liver, kidney, adrenal gland, urinary bladder, spleen, pancreas, thymus, thyroid gland, parathyroid gland, trachea, esophagus, lung, heart, salivary gland, lymph node, stomach, duodenum, jejunum, ileum, colon, rectum, preputial gland, clitoral gland, skin, brain, pituitary gland, bone marrow, prostate, seminal vesicle, ovary, uterus, and vagina were examined macroscopically and fixed in $10 \%$ neutral buffered formalin for histopathological examination. Testis and eyes with Harderian glands were also fixed with Bouin's solution and Davidson solution, respectively. The nasal cavity and femora were decalcified for up to 3 weeks. Formalin-fixed samples were embedded in paraffin, sectioned, and stained with hematoxylin-eosin for histological analysis using a light microscope (IX61, Olympus, Tokyo, Japan).

\section{Statistical analysis}

Data of male and female rats were analyzed separately by one-way analysis of variance (ANOVA) followed by multiple comparison with Dunnett's test using SPSS software version 19 (SPSS Inc., Chicago, IL, USA). Statistical significance level was set at $p<0.05$.

\section{Results}

\section{Single oral dose toxicity study}

In the 14-day observation of acute toxicity test using rats following a single oral administration of TS extract at 125, $250,500,1000$, or $2000 \mathrm{mg} / \mathrm{kg}$, no significant change in body weights attributable to the administration of TS extract was observed (Fig. 1). There were no significant changes in clinical signs, mortalities, or macroscopic necropsy examination of any organs at postmortem in either male or female rats following the administration of TS extract at any dose tested (data not shown).

\section{4-day repeat-dose oral toxicity study}

Repeated administrations of TS extract at 125, 250, 500, 1000 , and $2000 \mathrm{mg} / \mathrm{kg}$ by gavage for 14 days caused some side effects. As shown in Additional file 1, one male rat in the group treated with TS extract at $1000 \mathrm{mg} / \mathrm{kg}$ died at 13 days, showing clinical symptoms such as diarrhea and epistaxis. One male and two females in the group treated with TS extract at $2000 \mathrm{mg} /$ $\mathrm{kg}$ also died at 7 days and 10 days, respectively, showing severe diarrhea and epistaxis. Although two male rats treated with $500 \mathrm{mg} / \mathrm{kg}$ of TS extract showed diarrhea at 6 days, they recovered within $12 \mathrm{~h}$ after symptom onset. Body weights of male rats in 1000 and $2000 \mathrm{mg} / \mathrm{kg}$ of TS extract treatment groups were significantly decreased compared to those in the negative control group at 7 days (Fig. 2). At the end of the 14-day observation period, macroscopic examination revealed no TS extract-related abnormality at necropsy in either sex of rats, although spontaneous lesions, such as congestion in lung and thymus, were sporadically observed without showing dose-dependency (Additional file 2). Absolute liver weights of male rats treated with TS extract at dose of $500 \mathrm{mg} / \mathrm{kg}(6.844 \pm 0.820 \mathrm{~g}), 1000 \mathrm{mg} / \mathrm{kg}(6.983 \pm 1.385 \mathrm{~g})$, or $2000 \mathrm{mg} / \mathrm{kg}(7.486 \pm 0.662 \mathrm{~g})$ were significant decreased compared to those of negative control groups (8.716 \pm 


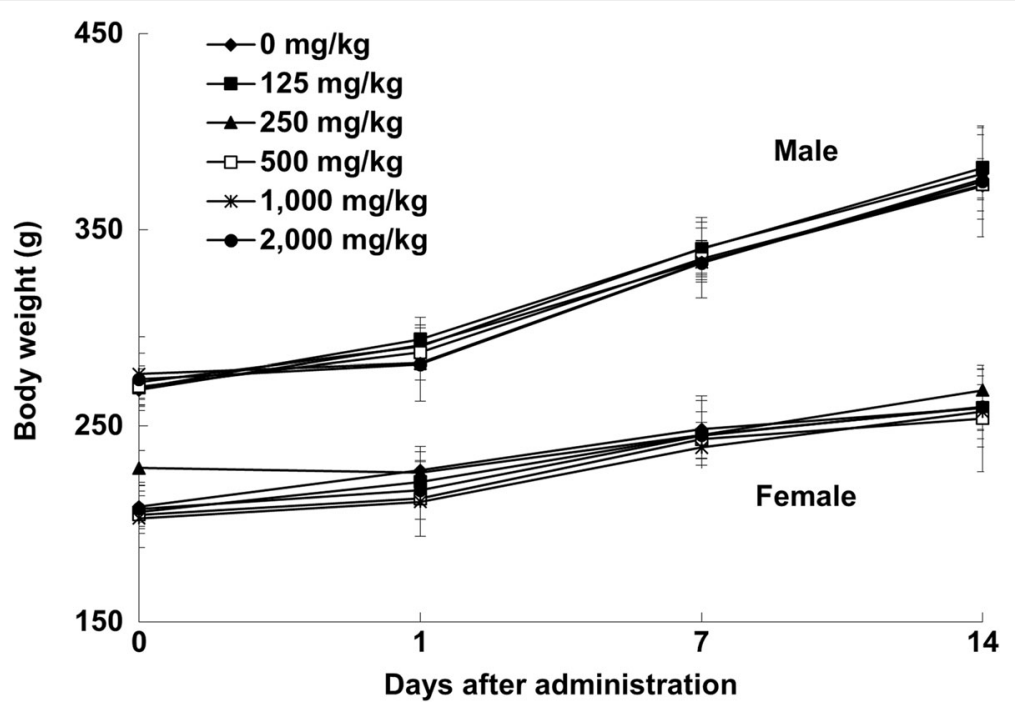

Fig. 1 Body weight values of male and female SD rats orally administered with Tiglium seed extract in single dose toxicity study. Data are expressed as means $\pm \mathrm{SD}$ ( $n=3 /$ sex/group)

0.379 g) (Additional file 3). Similarly, female rats treated with TS extract at dose of $500 \mathrm{mg} / \mathrm{kg}(4.618 \pm 0.268 \mathrm{~g})$ or $1000 \mathrm{mg} / \mathrm{kg}(4.263 \pm 0.205 \mathrm{~g})$ showed significant decreases in absolute liver weights in comparison with those of negative control groups $(5.560 \pm 0.389 \mathrm{~g})$. Relative liver weights of male rats treated with $500 \mathrm{mg} / \mathrm{kg}$ (3.860 \pm $0.202 \%)$ or $1000 \mathrm{mg} / \mathrm{kg}(3.876 \pm 0.200 \%)$ of TS extract were significantly lower than those of the negative control group $(4.312 \pm 0.177 \%)$. Relative weights of liver in female rats treated with $500 \mathrm{mg} / \mathrm{kg}(3.530 \pm 0.150 \%), 1000 \mathrm{mg} / \mathrm{kg}$ $(3.399 \pm 0.140 \%)$, or $2000 \mathrm{mg} / \mathrm{kg}(4.446 \pm 0.243 \%)$ of TS extract were also significantly changed compared with those in the negative control group (4.082 $\pm 0.178 \%)$. Of five TS extract concentrations tested, $500 \mathrm{mg} / \mathrm{kg}$ was selected as the highest dose of TS extract for 13-week repeat-dose oral toxicity study.

\section{3-week repeat-dose oral toxicity study}

General observation, body weight, feed intake, and water consumption

During the study period for the subchronic toxicity trial, one female rat treated with TS extract at $125 \mathrm{mg} / \mathrm{kg}$ died

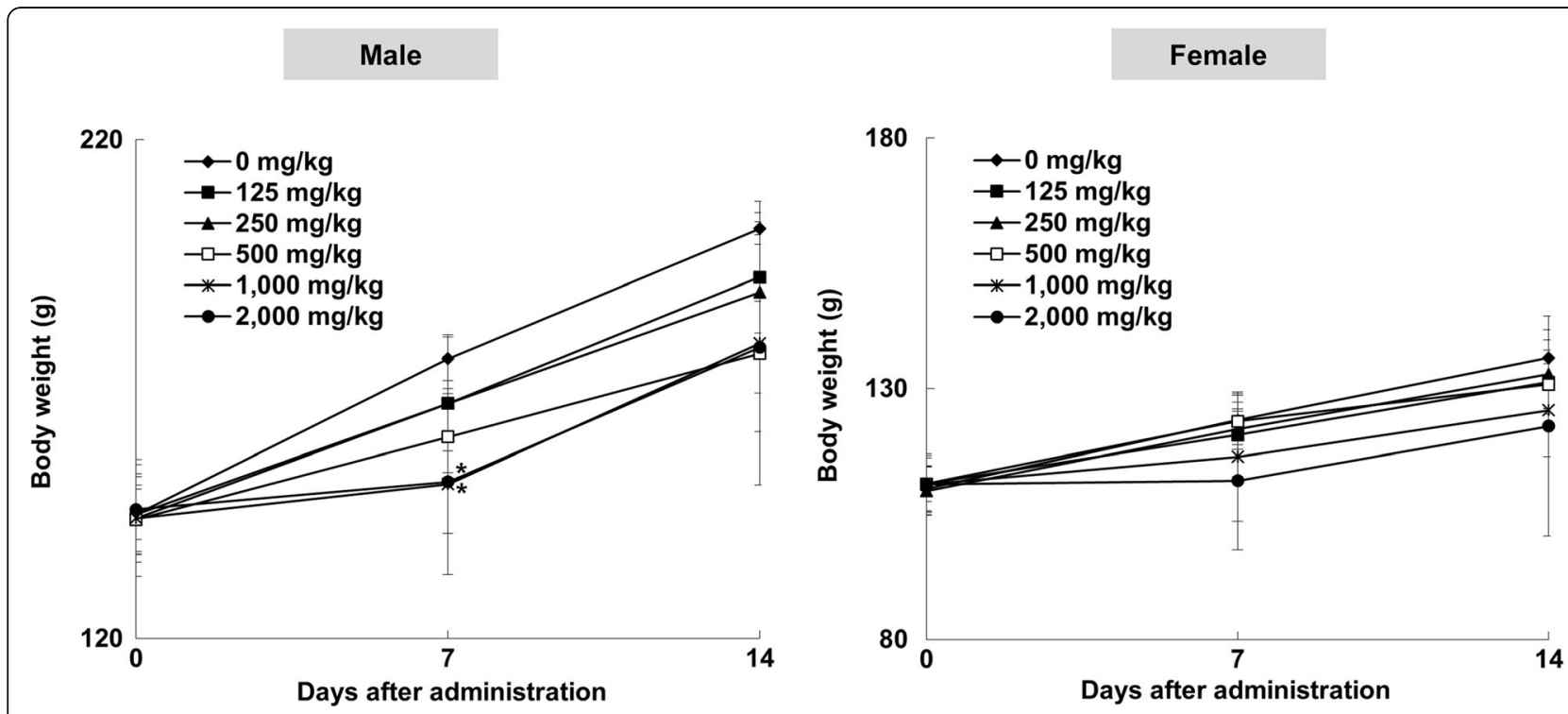

Fig. 2 Body weight values of male and female F344 rats orally administered with Tiglium seed extract in 14-day repeat-dose toxicity study. Data are expressed as means \pm SD ( $n=3-5 /$ sex/group). * Significantly different from Control group $(p<0.05)$ 
at 39 days after administration. However, the death for this rat was not considered to be TS extract-related because gross and histological lesions were transient without showing dose-dependency. In clinical observation for exterior appearances and behavior, no adverse clinical signs attributable to TS extract were noted at any dose tested in males or females. Likewise, TS extract did not affect body weight gains at any dose tested throughout the study period (Fig. 3). Although statistically significant change in daily food intake of female rats receiving TS extract at $500 \mathrm{mg} / \mathrm{kg}$ was observed at 13 weeks (Additional file 4A), such change was considered as incidental since this was sporadic and minimal. There were no significant changes in water consumption for 13 weeks after TS extract administration among groups in either males or females (Additional file 4B).

\section{Hematology and serum biochemistry}

Results of hematological analysis are shown in Table 1. PLT levels in males treated with TS extract at dose of $250 \mathrm{mg} / \mathrm{kg}(614 \pm 63.5)$ were significantly increased compared to those in the control group $(528 \pm 48.9)$. However, this finding was not regarded to be toxicologically relevant because this occurred infrequently without showing consistency between sexes.

Serum biochemical analysis revealed that TC levels in females following TS extract treatment at $125 \mathrm{mg} / \mathrm{kg}(85$ $\pm 8.9 \mathrm{mg} / \mathrm{dL}), 250 \mathrm{mg} / \mathrm{kg}(84 \pm 7.2 \mathrm{mg} / \mathrm{dL})$, or $500 \mathrm{mg} / \mathrm{kg}$ $(81 \pm 7.1 \mathrm{mg} / \mathrm{dL})$ were significantly decreased than those of the negative control group $(95 \pm 7.4 \mathrm{mg} / \mathrm{dL}$ ) (Table 2). Serum levels of TP were in rats treated with TS extract at
$62.5 \mathrm{mg} / \mathrm{kg}$ (male: $7.1 \pm 0.19 \mathrm{~g} / \mathrm{dL}$ ) or $125 \mathrm{mg} / \mathrm{kg}$ (male: $7.2 \pm 0.25 \mathrm{~g} / \mathrm{dL}$; female: $6.2 \pm 0.29 \mathrm{~g} / \mathrm{dL}$ ) were significantly changed compared to their respective negative control groups (male: $6.8 \pm 0.18 \mathrm{~g} / \mathrm{dL}$; female: $6.5 \pm 0.32 \mathrm{~g} / \mathrm{dL}$ ). Albumin levels were significantly increased in the group treated with $62.5 \mathrm{mg} / \mathrm{kg}$ of TS extract $(3.2 \pm 0.10 \mathrm{~g} / \mathrm{dL})$ in males but decreased in the group treated with $125 \mathrm{mg} / \mathrm{kg}$ of TS extract $(2.8 \pm 0.12 \mathrm{~g} / \mathrm{dL})$ in females compared with those in their respective negative control groups (male: $3.0 \pm 0.05 \mathrm{~g} / \mathrm{dL}$; female: $3.0 \pm 0.15 \mathrm{~g} / \mathrm{dL}$ ). Serum AST levels in the group treated with $500 \mathrm{mg} / \mathrm{kg}$ of TS extract (125 \pm $27.2 \mathrm{IU} / \mathrm{L})$ were significantly lower than those of the negative control group $(172 \pm 51.0 \mathrm{IU} / \mathrm{L})$ in males. Serum levels of ALT after subchronic administration of TS extract at $500 \mathrm{mg} / \mathrm{kg}$ in females $(55 \pm 7.0 \mathrm{IU} / \mathrm{L})$ were significantly decreased in comparison with those in the negative control group $(71 \pm 17.3 \mathrm{IU} / \mathrm{L})$. Levels of TG in groups treated with TS extract at $125 \mathrm{mg} / \mathrm{kg}(59 \pm 15.6 \mathrm{mg} / \mathrm{dL})$ or $250 \mathrm{mg} / \mathrm{kg}(60 \pm 10.4 \mathrm{mg} / \mathrm{dL})$ were also significantly lower than those of the negative control group $(81 \pm 28.4 \mathrm{mg}$ / $\mathrm{dL}$ ) in females.

\section{Organ weights and histopathological changes}

Results of absolute and relative organ weights are shown in Table 3. Relative weights of testis were significantly increased in males treated with TS extract at $500 \mathrm{mg} / \mathrm{kg}$ $(0.471 \pm 0.036 \%)$ compared to those of the negative control group $(0.426 \pm 0.023 \%)$. Absolute weights of thymus in male rats treated with TS extract at $250 \mathrm{mg} / \mathrm{kg}(0.198$ $\pm 0.017 \mathrm{~g})$ or $500 \mathrm{mg} / \mathrm{kg}(0.190 \pm 0.024 \mathrm{~g})$ were significantly lower than those in the negative control group

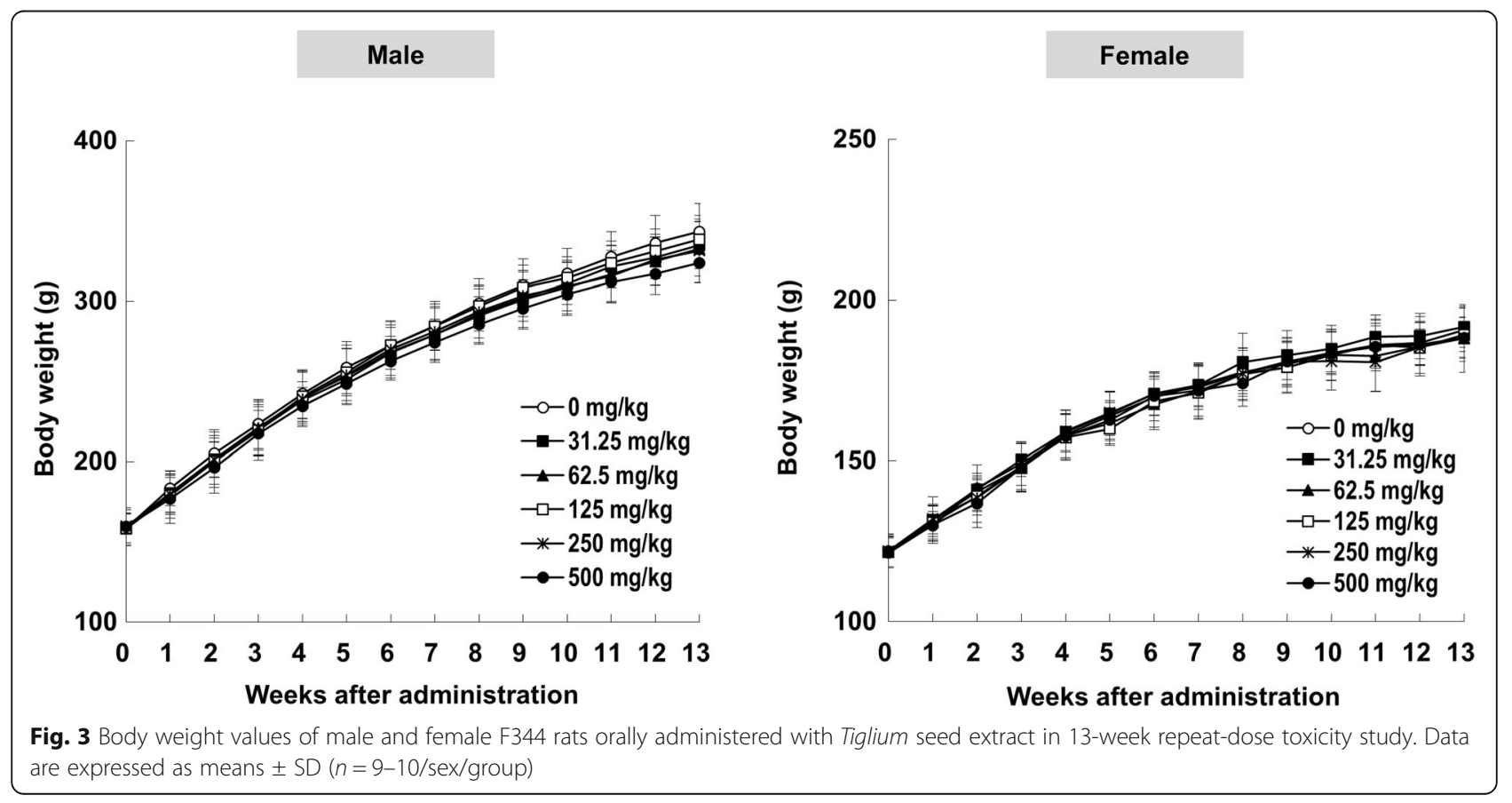


Table 1 Hematological data of male and female F344 rats orally administered with Tiglium seed extract for 13 weeks

\begin{tabular}{|c|c|c|c|c|c|c|}
\hline & \multicolumn{6}{|c|}{ Dose of Tiglium seed (mg/kg) } \\
\hline & $0^{\mathrm{a}}$ & 31.25 & 62.5 & 125 & 250 & 500 \\
\hline \multicolumn{7}{|l|}{ Males } \\
\hline WBC $\left(10^{3} / \mathrm{mm}^{3}\right)$ & $6.1 \pm 1.28$ & $5.9 \pm 0.90$ & $6.5 \pm 1.16$ & $6.9 \pm 1.37$ & $6.4 \pm 1.49$ & $5.9 \pm 1.60$ \\
\hline $\mathrm{RBC}\left(10^{6} / \mathrm{mm}^{3}\right)$ & $8.46 \pm 0.475$ & $8.58 \pm 0.282$ & $8.52 \pm 0.271$ & $8.53 \pm 0.351$ & $8.69 \pm 0.431$ & $8.53 \pm 0.279$ \\
\hline $\mathrm{HGB}(\mathrm{g} / \mathrm{dl})$ & $14.1 \pm 0.36$ & $14.2 \pm 0.26$ & $14.2 \pm 0.21$ & $14.2 \pm 0.37$ & $14.4 \pm 0.49$ & $14.3 \pm 0.40$ \\
\hline $\mathrm{HCT}(\%)$ & $39.0 \pm 2.10$ & $39.4 \pm 1.31$ & $39.2 \pm 1.37$ & $39.6 \pm 1.58$ & $40.2 \pm 2.10$ & $39.6 \pm 1.45$ \\
\hline $\operatorname{PLT}\left(10^{3} / \mathrm{mm}^{3}\right)$ & $528 \pm 48.9$ & $565 \pm 45.5$ & $568 \pm 37.2$ & $556 \pm 69.5$ & $614 \pm 63.5^{*}$ & $595 \pm 36.4$ \\
\hline MCV (fl) & $46 \pm 0.7$ & $46 \pm 0.5$ & $46 \pm 0.4$ & $46 \pm 0.5$ & $46 \pm 0.7$ & $46 \pm 0.5$ \\
\hline $\mathrm{MCH}(\mathrm{pg})$ & $16.7 \pm 0.57$ & $16.5 \pm 0.42$ & $16.7 \pm 0.43$ & $16.7 \pm 0.42$ & $16.6 \pm 0.45$ & $16.7 \pm 0.43$ \\
\hline $\mathrm{MCHC}(\mathrm{g} / \mathrm{dl})$ & $36.3 \pm 1.20$ & $36.0 \pm 0.87$ & $36.2 \pm 0.96$ & $35.9 \pm 0.77$ & $35.9 \pm 0.88$ & $36.1 \pm 1.04$ \\
\hline Reticulocytes (/100 RBC) & $1.3 \pm 0.54$ & $1.1 \pm 0.48$ & $1.3 \pm 0.31$ & $1.3 \pm 0.43$ & $1.2 \pm 0.37$ & $1.1 \pm 0.52$ \\
\hline Neutrophils (\%) & $22 \pm 7.0$ & $19 \pm 6.4$ & $20 \pm 5.0$ & $20 \pm 7.9$ & $21 \pm 7.4$ & $23 \pm 5.7$ \\
\hline Eosinophils (\%) & $0 \pm 0.4$ & $1 \pm 1.0$ & $1 \pm 0.6$ & $0 \pm 0.7$ & $1 \pm 0.7$ & $1 \pm 1.1$ \\
\hline Basophils (\%) & $0 \pm 0.0$ & $0 \pm 0.0$ & $0 \pm 0.0$ & $0 \pm 0.0$ & $0 \pm 0.0$ & $0 \pm 0.0$ \\
\hline Lymphocytes (\%) & $77 \pm 7.2$ & $80 \pm 6.9$ & $79 \pm 4.8$ & $79 \pm 8.0$ & $78 \pm 7.8$ & $76 \pm 5.9$ \\
\hline Monocytes (\%) & $1 \pm 1.1$ & $1 \pm 0.8$ & $1 \pm 1.0$ & $1 \pm 1.0$ & $1 \pm 0.7$ & $1 \pm 0.7$ \\
\hline \multicolumn{7}{|l|}{ Females } \\
\hline WBC $\left(10^{3} / \mathrm{mm}^{3}\right)$ & $5.3 \pm 0.92$ & $4.9 \pm 0.63$ & $5.1 \pm 0.69$ & $5.1 \pm 0.82$ & $5.0 \pm 1.23$ & $4.3 \pm 0.77$ \\
\hline $\mathrm{RBC}\left(10^{6} / \mathrm{mm}^{3}\right)$ & $7.59 \pm 0.380$ & $7.55 \pm 0.369$ & $7.49 \pm 0.346$ & $7.67 \pm 0.326$ & $7.68 \pm 0.309$ & $7.48 \pm 0.293$ \\
\hline $\mathrm{HGB}(\mathrm{g} / \mathrm{dl})$ & $13.9 \pm 0.40$ & $14.0 \pm 0.37$ & $13.8 \pm 0.27$ & $14.1 \pm 0.40$ & $14.2 \pm 0.33$ & $13.8 \pm 0.24$ \\
\hline HCT (\%) & $37.3 \pm 1.55$ & $37.4 \pm 1.64$ & $37.0 \pm 1.44$ & $37.9 \pm 1.50$ & $38.1 \pm 1.33$ & $37.1 \pm 1.06$ \\
\hline $\operatorname{PLT}\left(10^{3} / \mathrm{mm}^{3}\right)$ & $625 \pm 47.7$ & $623 \pm 27.2$ & $620 \pm 51.8$ & $643 \pm 60.8$ & $649 \pm 47.3$ & $638 \pm 81.4$ \\
\hline $\mathrm{MCV}(\mathrm{fl})$ & $49 \pm 0.5$ & $49 \pm 0.7$ & $50 \pm 0.5$ & $49 \pm 0.4$ & $50 \pm 0.7$ & $50 \pm 0.7$ \\
\hline $\mathrm{MCH}(\mathrm{pg})$ & $18.3 \pm 0.57$ & $18.5 \pm 0.53$ & $18.4 \pm 0.53$ & $18.4 \pm 0.50$ & $18.5 \pm 0.49$ & $18.5 \pm 0.65$ \\
\hline $\mathrm{MCHC}(\mathrm{g} / \mathrm{dl})$ & $37.2 \pm 0.75$ & $37.4 \pm 0.86$ & $37.2 \pm 0.76$ & $37.2 \pm 0.84$ & $37.2 \pm 0.77$ & $37.3 \pm 0.91$ \\
\hline Reticulocytes (/100 RBC) & $1.0 \pm 0.25$ & $1.4 \pm 0.39$ & $1.3 \pm 0.41$ & $1.2 \pm 0.27$ & $1.1 \pm 0.43$ & $1.3 \pm 0.53$ \\
\hline Neutrophils (\%) & $17 \pm 6.0$ & $13 \pm 3.6$ & $18 \pm 4.2$ & $16 \pm 7.7$ & $17 \pm 5.6$ & $20 \pm 4.6$ \\
\hline Eosinophils (\%) & $1 \pm 1.0$ & $1 \pm 0.7$ & $1 \pm 0.6$ & $1 \pm 1.2$ & $0 \pm 0.3$ & $1 \pm 0.7$ \\
\hline Basophils (\%) & $0 \pm 0.0$ & $0 \pm 0.0$ & $0 \pm 0.0$ & $0 \pm 0.0$ & $0 \pm 0.0$ & $0 \pm 0.0$ \\
\hline Lymphocytes (\%) & $81 \pm 5.9$ & $86 \pm 4.2$ & $81 \pm 3.9$ & $82 \pm 7.1$ & $82 \pm 6.4$ & $79 \pm 4.8$ \\
\hline Monocytes (\%) & $1 \pm 1.1$ & $1 \pm 0.5$ & $1 \pm 0.7$ & $1 \pm 1.4$ & $1 \pm 0.9$ & $1 \pm 1.2$ \\
\hline
\end{tabular}

Data expressed as means \pm SD

*Significantly different from Control group $(p<0.05)$

${ }^{a}$ Control group

$(0.228 \pm 0.011 \mathrm{~g})$. Relative heart weights of male rats treated with TS extract at dose of $500 \mathrm{mg} / \mathrm{kg}(0.295 \pm$ $0.009 \%$ ) were significant higher than those of the negative control group $(0.282 \pm 0.010 \%)$.

In gross visual observation, organs of rats in TS extract treatment groups at all dose levels tested showed macroscopic pathologies similar to those of control rats (Additional file 5). Histopathological evaluation did not reveal dose-related abnormal symptoms in sampled organs or tissues of TS extract groups, although spontaneous lesions, including basophilic tubule in kidney (six out of 10 male control rats) and focal inflammation in heart (nine out of 10 male control rats) encountered more frequently in male control rats, occurred in several tissues of rats in both control and TS extract groups (Table 4).

\section{Discussion}

TS is an important traditional medicine for treating constipation, dyspepsia, and dysenteria [8-11]. Mature Croton tiglium contains several anti-cancer components, including croton alkaloid, flavonoids, and diterpenes [30-32]. Croton oil from seeds of Croton tiglium is known to exert a variety of remarkable biological activities, including 
Table 2 Serum biochemistry data of male and female F344 rats orally administered with Tiglium seed extract for 13 weeks

\begin{tabular}{|c|c|c|c|c|c|c|}
\hline & \multicolumn{6}{|c|}{ Dose of Tiglium seed (mg/kg) } \\
\hline & $\overline{0^{a}}$ & 31.25 & 62.5 & 125 & 250 & 500 \\
\hline \multicolumn{7}{|l|}{ Males } \\
\hline BUN (mg/dL) & $22 \pm 3.0$ & $23 \pm 3.7$ & $24 \pm 4.6$ & $23 \pm 3.8$ & $22 \pm 4.2$ & $22 \pm 4.0$ \\
\hline $\mathrm{TC}(\mathrm{mg} / \mathrm{dL})$ & $71 \pm 7.2$ & $74 \pm 5.7$ & $73 \pm 7.3$ & $77 \pm 5.8$ & $69 \pm 6.2$ & $69 \pm 6.8$ \\
\hline $\mathrm{TP}(\mathrm{g} / \mathrm{dL})$ & $6.8 \pm 0.18$ & $7.0 \pm 0.19$ & $7.1 \pm 0.19^{*}$ & $7.2 \pm 0.25^{*}$ & $6.8 \pm 0.20$ & $6.8 \pm 0.13$ \\
\hline Albumin (g/dL) & $3.0 \pm 0.05$ & $3.1 \pm 0.09$ & $3.2 \pm 0.10^{*}$ & $3.2 \pm 0.16$ & $3.1 \pm 0.10$ & $3.1 \pm 0.08$ \\
\hline TB (mg/dL) & $0.1 \pm 0.00$ & $0.1 \pm 0.00$ & $0.1 \pm 0.00$ & $0.1 \pm 0.00$ & $0.1 \pm 0.00$ & $0.1 \pm 0.00$ \\
\hline ALP (IU/L) & $207 \pm 22.8$ & $219 \pm 43.4$ & $210 \pm 26.1$ & $201 \pm 33.2$ & $188 \pm 22.3$ & $189 \pm 27.3$ \\
\hline AST (IU/L) & $172 \pm 51.0$ & $150 \pm 29.5$ & $161 \pm 32.6$ & $161 \pm 31.8$ & $132 \pm 34.1$ & $125 \pm 27.2^{*}$ \\
\hline ALT (IU/L) & $83 \pm 22.4$ & $70 \pm 10.5$ & $75 \pm 12.2$ & $81 \pm 11.5$ & $74 \pm 11.8$ & $67 \pm 7.8$ \\
\hline Creatinine (mg/dL) & $0.6 \pm 0.05$ & $0.5 \pm 0.05$ & $0.6 \pm 0.07$ & $0.6 \pm 0.07$ & $0.5 \pm 0.05$ & $0.6 \pm 0.05$ \\
\hline $\mathrm{TG}(\mathrm{mg} / \mathrm{dL})$ & $194 \pm 39.2$ & $210 \pm 35.8$ & $178 \pm 38.3$ & $221 \pm 82.7$ & $180 \pm 55.4$ & $151 \pm 40.7$ \\
\hline \multicolumn{7}{|l|}{ Females } \\
\hline BUN (mg/dL) & $22 \pm 5.5$ & $21 \pm 4.7$ & $20 \pm 3.9$ & $19 \pm 3.4$ & $20 \pm 4.4$ & $18 \pm 3.0$ \\
\hline $\mathrm{TC}(\mathrm{mg} / \mathrm{dL})$ & $95 \pm 7.4$ & $93 \pm 10.6$ & $93 \pm 8.5$ & $85 \pm 8.9^{*}$ & $84 \pm 7.2^{*}$ & $81 \pm 7.1^{*}$ \\
\hline $\mathrm{TP}(\mathrm{g} / \mathrm{dL})$ & $6.5 \pm 0.32$ & $6.6 \pm 0.22$ & $6.6 \pm 0.15$ & $6.2 \pm 0.29^{*}$ & $6.3 \pm 0.20$ & $6.4 \pm 0.21$ \\
\hline Albumin (g/dL) & $3.0 \pm 0.15$ & $3.0 \pm 0.11$ & $3.0 \pm 0.08$ & $2.8 \pm 0.12^{*}$ & $2.9 \pm 0.09$ & $2.9 \pm 0.12$ \\
\hline TB (mg/dL) & $0.1 \pm 0.00$ & $0.1 \pm 0.00$ & $0.1 \pm 0.00$ & $0.1 \pm 0.00$ & $0.1 \pm 0.00$ & $0.1 \pm 0.00$ \\
\hline ALP (IU/L) & $176 \pm 21.1$ & $187 \pm 19.9$ & $199 \pm 39.0$ & $184 \pm 32.7$ & $182 \pm 30.5$ & $166 \pm 27.9$ \\
\hline AST (IU/L) & $148 \pm 35.1$ & $152 \pm 30.9$ & $150 \pm 29.4$ & $134 \pm 26.9$ & $143 \pm 33.8$ & $137 \pm 32.2$ \\
\hline $\mathrm{ALT}(\mathrm{IU} / \mathrm{L})$ & $71 \pm 17.3$ & $70 \pm 13.8$ & $66 \pm 6.9$ & $63 \pm 10.0$ & $63 \pm 9.1$ & $55 \pm 7.0^{*}$ \\
\hline Creatinine (mg/dL) & $0.6 \pm 0.10$ & $0.6 \pm 0.07$ & $0.6 \pm 0.05$ & $0.6 \pm 0.07$ & $0.7 \pm 0.07$ & $0.6 \pm 0.04$ \\
\hline $\mathrm{TG}(\mathrm{mg} / \mathrm{dL})$ & $81 \pm 28.4$ & $66 \pm 11.5$ & $78 \pm 22.0$ & $59 \pm 15.6^{*}$ & $60 \pm 10.4^{*}$ & $63 \pm 16.7$ \\
\hline
\end{tabular}

Data expressed as means \pm SD

*Significantly different from Control group $(p<0.05)$

${ }^{\text {aC }}$ ontrol group

purgative, analgesic, antimicrobial, and inflammatory properties $[8,11]$. It has also been particularly used for treating skin diseases including ringworm [33, 34]. Croton oil can modulate gastrointestinal motility and affect intestinal inflammation related to immunological milieu in mice [35]. Hu et al. [36] have also reported the effect of croton oil on spontaneous contraction of smooth muscle in rabbit jejunum. It has been shown that a pyrazine derivative crotonine isolated from leaves of Croton tiglium possesses analgesic effects [37].

In a previous study, data of in vitro chromosome aberration assay and in vivo micronucleus assay have demonstrated that TS extract has no clastogenic potential, although Ames test results have shown mutagenicity (base-substitution, frameshift, or cross-linking and oxidizing mutagen) of TS extract [38]. Genotoxicity, especially mutagenic potential, could be one initial risk factor closely involved in long-term carcinogenic pathway [39, 40]. Besides its mutagenicity, TS extract has also caused gap junctional intercellular communication (GJIC) dysfunction known to be involved in tumor promotion stage of carcinogenesis [38, 41]. Croton oil, one of main components of TS, contains many types of phorbol derivatives including 12-O-tetradecanoyl-phorbol-13-acetate (TPA) $[4,42]$ which act as a co-carcinogen or a tumor promoter [43], supporting the relationship between mutagenicity and GJIC inhibition of TS extract. Nonetheless, a comprehensive non-clinical subchronic toxicity study of TS extract under OECD guidelines and GLP regulation should be conducted for its safe use in humans.

In acute toxicity tests, single oral administration of TS extract at doses ranging from 0 to $2000 \mathrm{mg} / \mathrm{kg}$ showed no dose-related changes in mortality, body weights, or clinical signs, indicating that acute oral $\mathrm{LD}_{50}$ of TS extract was higher than $2000 \mathrm{mg} / \mathrm{kg}$ for both male and female rats. In 14 days of repeated-dose toxicity studies $(0$, $125,250,500,1000$, and $2000 \mathrm{mg} / \mathrm{kg} /$ day) conducted as a preliminary study for a 13-week repeated-dose toxicity study, four cases of death occurred in groups treated with TS extract at $1000 \mathrm{mg} / \mathrm{kg}$ and $2000 \mathrm{mg} / \mathrm{kg}$ (one male in $1000 \mathrm{mg} / \mathrm{kg}$ group, one male and two females in $2000 \mathrm{mg} / \mathrm{kg}$ group). Significant changes in parameters of body weights and organ weights were also observed in groups treated with TS extract at dose of $1000 \mathrm{mg} / \mathrm{kg}$ or 
Table 3 Organ weights of male and female F344 rats orally administered with Tiglium seed extract for 13 weeks

\begin{tabular}{|c|c|c|c|c|c|c|c|}
\hline & & \multicolumn{6}{|c|}{ Dose of Tiglium seed (mg/kg) } \\
\hline & & $\overline{0^{a}}$ & 31.25 & 62.5 & 125 & 250 & 500 \\
\hline \multicolumn{8}{|l|}{ Males } \\
\hline \multirow[t]{2}{*}{ Liver } & (g) & $10.564 \pm 0.488$ & $10.654 \pm 0.516$ & $10.171 \pm 0.727$ & $10.782 \pm 0.883$ & $10.378 \pm 0.862$ & $9.816 \pm 0.560$ \\
\hline & $(\% \mathrm{BW})$ & $3.188 \pm 0.109$ & $3.303 \pm 0.162$ & $3.195 \pm 0.144$ & $3.331 \pm 0.204$ & $3.301 \pm 0.226$ & $3.216 \pm 0.179$ \\
\hline \multirow[t]{2}{*}{ Kidney } & (g) & $0.991 \pm 0.023$ & $0.981 \pm 0.044$ & $0.957 \pm 0.063$ & $0.987 \pm 0.066$ & $0.965 \pm 0.087$ & $0.934 \pm 0.049$ \\
\hline & $(\% \mathrm{BW})$ & $0.300 \pm 0.015$ & $0.304 \pm 0.017$ & $0.301 \pm 0.016$ & $0.305 \pm 0.012$ & $0.307 \pm 0.015$ & $0.306 \pm 0.012$ \\
\hline \multirow[t]{2}{*}{ Testis } & (g) & $1.408 \pm 0.050$ & $1.420 \pm 0.066$ & $1.396 \pm 0.066$ & $1.439 \pm 0.075$ & $1.426 \pm 0.060$ & $1.439 \pm 0.119$ \\
\hline & $(\% \mathrm{BW})$ & $0.426 \pm 0.023$ & $0.440 \pm 0.015$ & $0.439 \pm 0.023$ & $0.445 \pm 0.024$ & $0.454 \pm 0.024$ & $0.471 \pm 0.036^{*}$ \\
\hline \multirow[t]{2}{*}{ Thymus } & (g) & $0.228 \pm 0.011$ & $0.231 \pm 0.025$ & $0.209 \pm 0.027$ & $0.206 \pm 0.013$ & $0.198 \pm 0.017^{*}$ & $0.190 \pm 0.024^{*}$ \\
\hline & $(\% \mathrm{BW})$ & $0.069 \pm 0.005$ & $0.071 \pm 0.005$ & $0.066 \pm 0.010$ & $0.064 \pm 0.004$ & $0.063 \pm 0.003$ & $0.062 \pm 0.008$ \\
\hline \multirow[t]{2}{*}{ Heart } & (g) & $0.937 \pm 0.058$ & $0.915 \pm 0.039$ & $0.933 \pm 0.063$ & $0.922 \pm 0.049$ & $0.910 \pm 0.051$ & $0.902 \pm 0.047$ \\
\hline & (\%BW) & $0.282 \pm 0.010$ & $0.283 \pm 0.008$ & $0.293 \pm 0.009$ & $0.285 \pm 0.010$ & $0.289 \pm 0.007$ & $0.295 \pm 0.009^{*}$ \\
\hline \multirow[t]{2}{*}{ Lung } & (g) & $1.234 \pm 0.050$ & $1.171 \pm 0.069$ & $1.181 \pm 0.063$ & $1.216 \pm 0.125$ & $1.181 \pm 0.100$ & $1.197 \pm 0.071$ \\
\hline & (\%BW) & $0.373 \pm 0.016$ & $0.363 \pm 0.019$ & $0.371 \pm 0.016$ & $0.377 \pm 0.051$ & $0.376 \pm 0.022$ & $0.392 \pm 0.026$ \\
\hline \multicolumn{8}{|l|}{ Females } \\
\hline \multirow[t]{2}{*}{ Liver } & (g) & $5.176 \pm 0.348$ & $5.293 \pm 0.150$ & $5.257 \pm 0.249$ & $5.212 \pm 0.189$ & $5.126 \pm 0.350$ & $5.152 \pm 0.204$ \\
\hline & (\%BW) & $2.844 \pm 0.193$ & $2.872 \pm 0.090$ & $2.917 \pm 0.192$ & $2.887 \pm 0.160$ & $2.849 \pm 0.168$ & $2.885 \pm 0.106$ \\
\hline \multirow[t]{2}{*}{ Kidney } & (g) & $0.578 \pm 0.039$ & $0.580 \pm 0.027$ & $0.586 \pm 0.026$ & $0.577 \pm 0.030$ & $0.581 \pm 0.034$ & $0.586 \pm 0.022$ \\
\hline & $(\% \mathrm{BW})$ & $0.317 \pm 0.019$ & $0.315 \pm 0.016$ & $0.324 \pm 0.009$ & $0.319 \pm 0.012$ & $0.323 \pm 0.016$ & $0.328 \pm 0.010$ \\
\hline \multirow[t]{2}{*}{ Thymus } & (g) & $0.191 \pm 0.008$ & $0.192 \pm 0.014$ & $0.189 \pm 0.014$ & $0.193 \pm 0.024$ & $0.184 \pm 0.024$ & $0.185 \pm 0.016$ \\
\hline & (\%BW) & $0.105 \pm 0.004$ & $0.104 \pm 0.006$ & $0.105 \pm 0.007$ & $0.107 \pm 0.015$ & $0.102 \pm 0.012$ & $0.103 \pm 0.009$ \\
\hline \multirow[t]{2}{*}{ Heart } & (g) & $0.579 \pm 0.026$ & $0.578 \pm 0.024$ & $0.575 \pm 0.029$ & $0.567 \pm 0.044$ & $0.562 \pm 0.030$ & $0.574 \pm 0.021$ \\
\hline & (\%BW) & $0.318 \pm 0.015$ & $0.314 \pm 0.008$ & $0.318 \pm 0.010$ & $0.313 \pm 0.014$ & $0.312 \pm 0.011$ & $0.321 \pm 0.008$ \\
\hline \multirow[t]{2}{*}{ Lung } & (g) & $0.923 \pm 0.112$ & $0.822 \pm 0.061$ & $0.851 \pm 0.055$ & $0.868 \pm 0.081$ & $0.854 \pm 0.036$ & $0.855 \pm 0.051$ \\
\hline & (\%BW) & $0.507 \pm 0.066$ & $0.446 \pm 0.031$ & $0.471 \pm 0.027$ & $0.481 \pm 0.055$ & $0.475 \pm 0.019$ & $0.479 \pm 0.037$ \\
\hline
\end{tabular}

Data expressed as means \pm SD

*Significantly different from Control group $(p<0.05)$

${ }^{\mathrm{a} C}$ Control group

more. Considering above results of meaningful toxicity in 14-day repeat-dose oral toxicity study, $500 \mathrm{mg} / \mathrm{kg}$ was selected as the highest-dose group of TS extract for the 13-week repeated-dose toxicity study.

In the 13-week subchronic toxicity study, repeated administrations of TS extract at dose up to $500 \mathrm{mg} / \mathrm{kg}$ did not cause abnormal alterations in mortality or behavioral symptoms as early signs of toxicity. Rats treated with TS extract did not show any dose-associated difference in terms of body weight or food/water consumption either, indicating that TS extract did not retard animal growth or normal metabolism. Furthermore, results of our current analysis for hematological parameters associated with systemic toxic symptoms (i.e., HCT, HGB, PLT, $\mathrm{WBC}$, and $\mathrm{RBC}$ ) also indicated no adverse changes in either sex of rats after treatment with the highest dosage of TS extract.

Liver and kidney are major organs involved in the metabolism and elimination of drugs and other foreign compounds. They can be considered as major target organs that suffer systemic adverse reactions following oral administration of drugs [44, 45]. Serum concentrations of BUN and creatinine are the most widely used indicators in clinical biochemistry test for monitoring renal function $[46,47]$. Along with unchanged levels of serum biochemical parameters (BUN and creatinine) and kidney weights, pathology data (gross findings and histopathological findings) supported our conclusion that no renal toxicity could be attributed to TS extract treatment. The liver plays an important role in the metabolism, elimination, and detoxification of drugs or other agents [48]. Serum levels of four enzymes (ALT, AST, ALP, and TG) are commonly used as clinical biochemistry markers associated with liver damage. Among these enzymes, serum levels of ALP known to be increased in response to biliary obstruction [49] did not show any significant changes between negative control group and TS extract groups in male or female rats. Significant 


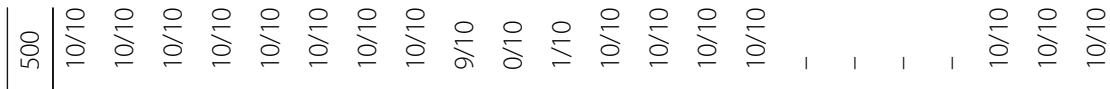

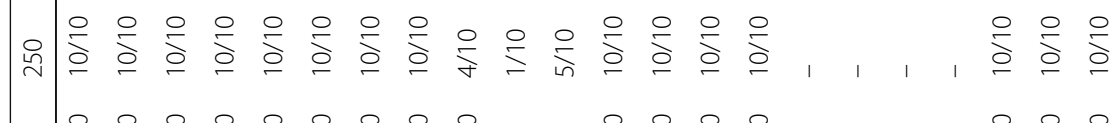

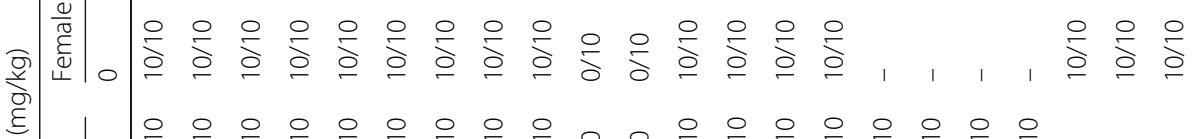

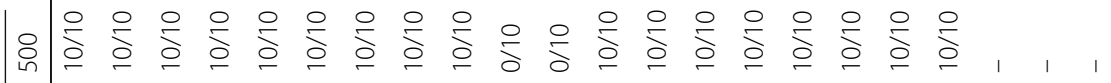

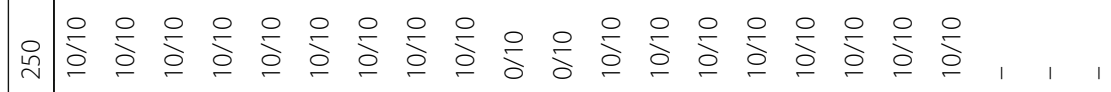

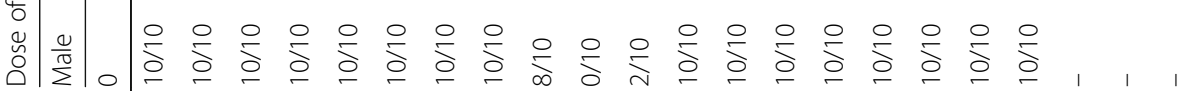

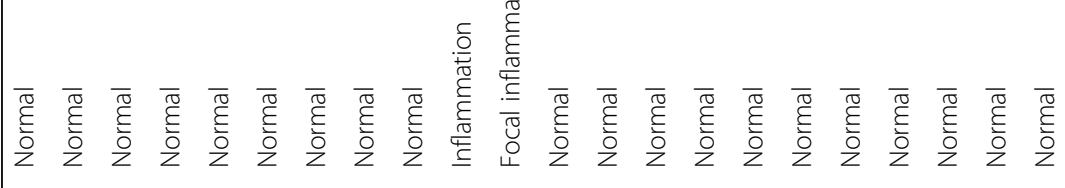

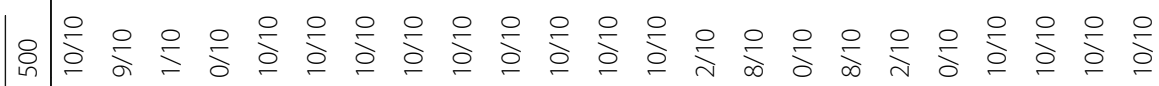

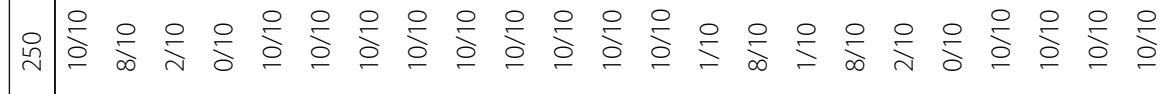

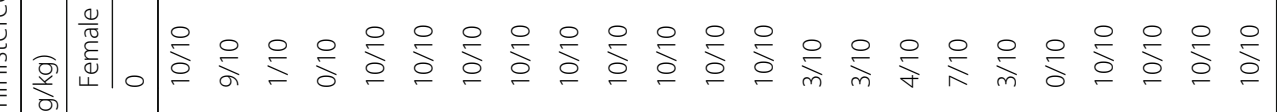

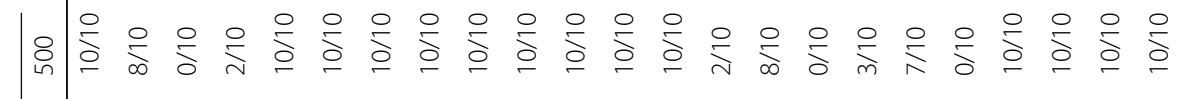

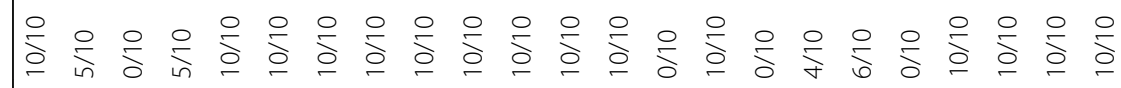


changes in other liver function markers such as ALT, AST (hepatocellular damage marker) [50, 51], and TG (fatty liver marker) [52] were observed in TS extract treatment groups, including decreased AST in males of $500 \mathrm{mg} / \mathrm{kg}$ group, decreased ALT in females of $500 \mathrm{mg} / \mathrm{kg}$ group, and decreased TG in females of $125 \mathrm{mg} / \mathrm{kg}$ and $250 \mathrm{mg} / \mathrm{kg}$ groups. However, these changes were considered incidental because they were within acceptable ranges (AST in males, $103 \pm 14.2 \mathrm{IU} / \mathrm{L}$; ALT in female, $52 \pm 11.7 \mathrm{IU} / \mathrm{L}$; TG in females, $65 \pm 34.3 \mathrm{mg} / \mathrm{dL}$; SLC, http://www.jslc.co.jp/ pdf/rat/005_F3442013.pdf). In addition, they were not sex- or dose-related. Combining organ weights with macroscopic/microscopic examination results, certain organ weights were significantly changed while no pathological (histopathological) changes in these organs were found, indicating that TS extract did not produce any toxic symptoms of internal major organs, including liver, kidney, lung, spleen, pancreas, or brain. Taken together, subchronic exposure of rats to TS extract exhibited no treatment-related changes at dose up to $500 \mathrm{mg} / \mathrm{kg}$. This corresponds to about $81 \mathrm{mg} / \mathrm{kg}$ in human by using the conversion help according to dosing adjustment guidelines of the US Food and Drug Administration [53].

\section{Conclusions}

Results of single and 13-week repeated oral dose toxicity studies clearly supported that administration of TS extract did not exert adverse effect in male or female rats for most toxicological factors. In particular, acute exposure to TS extract did not exert any significant toxic influence on rats at any dose tested, suggesting that oral $\mathrm{LD}_{50}$ of TS extract was greater than $2000 \mathrm{mg} / \mathrm{kg}$ for both sexes. Subchronic exposure of rats to TS extract did not induce treatmentrelated damage to target organs, indicating that subchronic NOAEL of TS extract was $500 \mathrm{mg} / \mathrm{kg}$ for both male and female rats. The current study is the first to provide important background on safety concerns of TS extract by analyzing the traditional acute toxicity and subchronic toxicity profile of TS extract comprehensively under OECD guidelines and GLP regulations for human safe consumption.

\section{Additional files}

Additional file 1: Survival of male and female F344 rats orally administered with Tiglium seed extract for 14 days. (TIF $630 \mathrm{~kb}$ )

Additional file 2: Summary incidence of gross findings of F344 rats orally administered with Tiglium seed extract for 14 days. (DOCX 17 kb)

Additional file 3: Organ weights of male and female F344 rats orally administered with Tiglium seed extract for 14 days. (DOCX $30 \mathrm{~kb}$ )

Additional file 4: Effects of Tiglium seed extract on the daily food intake and water consumption after oral administration in male and female rats for 13 weeks. (TIF 806 kb)

Additional file 5: Summary incidence of gross findings of F344 rats orally administered with Tiglium seed extract for 13 weeks. (DOCX 18 kb)

\section{Abbreviations}

ALP: Alkaline phosphatase; ALT: Alanine transaminase; AST: Aspartate transaminase; BUN: Blood urea nitrogen; GJIC: Gap junctional intercellular communication; GLP: Good Laboratory Practice; HCT: Hematocrit; HGB: Hemoglobin; LD 50 : Median lethal dose; MC: Methylcellulose; $\mathrm{MCH}$ : Mean corpuscular hemoglobin; MCHC: Mean corpuscular hemoglobin concentration; MCV: Mean corpuscular volume; NOAEL: No-observedadverse-effect-level; OECD: Organization for Economic Co-operation and Development; PLT: Platelet; RBC: Red blood cell; SD: Sprague-Dawley; TB: Total bilirubin; TC: Total cholesterol; TG: Triglyceride; TP: Total protein; TS: Tiglium seed; WBC: White blood cell

\section{Acknowledgements}

This work was supported by a grant from the Seoul National University Hospital research fund (09-2005-007-0) and a grant (MFDS234) from the Ministry of Food and Drug Safety.

\section{Funding}

A grant from the Seoul National University Hospital research fund (09-2005007-0) and a grant (MFDS234) from the Ministry of Food and Drug Safety supported this study. The funding body did not have any additional role in the study design, data collection and analysis, and manuscript preparation.

Availability of data and materials

The analyzed data supporting the conclusions of this article are included in this published article. The raw data are available from the corresponding author on reasonable request.

\section{Authors' contributions}

JWY and EK performed the biological experiments and wrote the manuscript. YSK, SHK, and JRY extracted and analyzed extracts. HCK, JSP, and JHC participated in the design of the study and provided technical advices. SKL and JJ conducted pathological analysis. HHK contributed to the analyzing the data, drafting and revising the manuscript. BCK participated in the design of the study, in discussions and reviewed the manuscript. All authors read and approved the final manuscript.

\section{Ethics approval}

All experimental procedures were approved by Institutional Animal Care and Use Committee of the Biomedical Research Institute at Seoul National University Hospital. The study protocol was designed in compliance with guidelines published by OECD as well as GLP regulations for Nonclinical Laboratory Studies of Korea Food Drug Administration.

\section{Consent for publication}

Not applicable.

\section{Competing interests}

The authors declare that they have no competing interests.

\section{Publisher's Note}

Springer Nature remains neutral with regard to jurisdictional claims in published maps and institutional affiliations.

\section{Author details}

${ }^{1}$ Department of Biotechnology, The Catholic University of Korea, 43 Jibongro, Bucheon 14662, Republic of Korea. ${ }^{2}$ Department of Experimental Animal Research, Biomedical Research Institute, Seoul National University Hospital, 101 Daehak-ro, Jongno-gu, Seoul 03080, Republic of Korea. ${ }^{3}$ Laboratory Animal Resource Center, Korea Research Institute of Bioscience and Biotechnology, 30 Yeongudanji-ro, Ochang-eup, Cheongwon-gu, Cheongju-si, Chungcheongbuk-do 28116, Republic of Korea. ${ }^{4}$ Biomedical Center for Animal Resource and Development, Seoul National University College of Medicine, 101 Daehak-ro, Jongno-gu, Seoul 03080, Republic of Korea. ${ }^{5}$ Department of Pathology, Seoul National University College of Medicine, 101 Daehak-ro, Jongno-gu, Seoul 03080, Republic of Korea. ${ }^{6}$ Department of Urology, Seoul National University College of Medicine, 101 Daehak-ro, Jongno-gu, Seoul 03080, Republic of Korea. ${ }^{7}$ Graduate School of Translational Medicine, Seoul National University College of Medicine, 101 Daehak-ro, Jongno-gu, Seoul 03080, Republic of Korea. ${ }^{8}$ Designed Animal and Transplantation Research Institute, Institute of GreenBio Science 
Technology, Seoul National University, 1447 Pyeongchang-daero, Daehwa-myeon, Pyeongchang-gun, Gangwon-do 25354, Republic of Korea. ${ }^{9}$ Graduate School of Translational Medicine, Seoul National University College of Medicine, 101 Daehak-ro, Jongno-gu, Seoul 110-744, Republic of Korea.

\section{Received: 18 June 2018 Accepted: 28 August 2018}

Published online: 12 September 2018

\section{References}

1. Zhang JT. Studies on the pharmacology and chemistry of traditional chinese and folk medicinal plants. Zhongguo Yi Xue Ke Xue Yuan Xue Bao. 1979;1:41-50.

2. Mortelmans K, Haworth S, Lawlor T, Speck W, Tainer B, Zeiger E. Salmonella mutagenicity tests: II. Results from the testing of 270 chemicals. Environ Mutagen. 1986:8:56-119.

3. Campos AR, Albuquerque FA, Rao VS, Maciel MA, Pinto AC. Investigations on the antinociceptive activity of crude extracts from Croton cajucara leaves in mice. Fitoterapia. 2002;73:116-20

4. Antonio S, Maria LFS, Giuseppina N. Traditional uses, chemistry and pharmacology of Croton species (Euphorbiaceae). J Braz Chem Soc. 2007:18:11-33.

5. Saputera, Mangunwidjaja D, Raharja S, Kardono LBS, Iswantini D. Characteristics, efficacy and safety testing of standardized extract of Croton tiglium seed from Indonesia as laxative material. Pak J Bio Sci. 2008;11:618-22.

6. Sandoval M, Okuhama NN, Clark M, Angeles FM, Lao J, Bustamante S, Miller MJ. Sangre de grado Croton palanostigma induces apoptosis in human gastrointestinal cancer cells. J Ethnopharmacol. 2002;80:121-9.

7. Lin HC, Kuo YL, Lee WJ, Yap HY, Wang SH. Antidermatophytic activity of Ethanolic extract from Croton tiglium. Biomed Res Int. 2016;2016:3237586.

8. Qiu HX. Flora of China. Beijing: Science Press; 1996.

9. Wang X, Lan M, Wu HP, Shi YQ, Lu J, Ding J, Wu KC, Jin JP, Fan DM. Direct effect of croton oil on intestinal epithelial cells and colonic smooth muscle cells. World J Gastroenterol. 2002:8:103-7.

10. Morimura K. The role of special group article in ancient Chinese medical prescription. Hist Sci (Tokyo). 2003;13:1-12

11. Tsai JC, Tsai S, Chang WC. Effect of ethanol extracts of three Chinese medicinal plants with laxative properties on ion transport of the rat intestinal epithelia. Biol Pharm Bull. 2004;27:162-5.

12. Hetter GP. An examination of the phenol-croton oil peel: part I. Dissecting the formula. Plast Reconstr Surg. 2000;105:227-39.

13. Song HK, Lee GS, Park SH, Noh EM, Kim JM, Ryu DG, Jung SH, Youn HJ, Lee YR, Kwon KB. Crotonis Fructus extract inhibits 12-O-Tetradecanoylphorbol13-acetate-induced expression of matrix Metalloproteinase-9 via the activator Protein-1 pathway in MCF-7 cells. J Breast Cancer. 2017;20:234-9.

14. Markman M. Safety issues in using complementary and alternative medicine. J Clin Oncol. 2002;20:39-41.

15. Dasgupta A. Review of abnormal laboratory test results and toxic effects due to use of herbal medicines. Am J Clin Pathol. 2003:120:127-37.

16. Jiang WY. Therapeutic wisdom in traditional Chinese medicine: a perspective from modern science. Trends Pharmacol Sci. 2005;26:558-63.

17. Bent $\mathrm{S}$. Herbal medicine in the United States: review of efficacy, safety, and regulation: grand rounds at University of California, san Francisco medical center. J Gen Intern Med. 2008;23:854-9.

18. Liu S, Yi LZ, Liang YZ. Traditional Chinese medicine and separation science. J Sep Sci. 2008:31:2113-37.

19. Liu IM, Tzeng TF, Liou SS, Chang CJ. Angelica acutiloba root alleviates advanced glycation end-product-mediated renal injury in streptozotocindiabetic rats. J Food Sci. 2011;76:H165-74.

20. Shin SH, Koo KH, Bae JS, Cha SB, Kang IS, Kang MS, Kim HS, Heo HS, Park MS, Gil GH, Lee JY, Kim KH, Li Y, Lee HK, Song SW, Choi HS, Kang BH, Kim JC. Single and 90-day repeated oral dose toxicity studies of fermented Rhus verniciflua stem bark extract in Sprague-Dawley rats. Food Chem Toxicol. 2013:55:617-26.

21. Che JH, Yun JW, Cho EY, Kim SH, Kim YS, Kim WH, Park JH, Son WC, Kim MK, Kang BC. Toxicologic assessment of Paecilomyces tenuipes in rats: renal toxicity and mutagenic potential. Regul Toxicol Pharmacol. 2014;70:527-34.

22. Che JH, Yun JW, Kim YS, Kim SH, You JR, Jang JJ, Kim HC, Kim HH, Kang BC. Genotoxicity and subchronic toxicity of Sophorae radix in rats: hepatotoxic and genotoxic potential. Regul Toxicol Pharmacol. 2015;71:379-87.

23. Yun JW, Kim SH, Kim YS, You JR, Kwon E, Jang JJ, Park IA, Kim HC, Kim HH, Che JH, Kang BC. Evaluation of subchronic (13 week) toxicity and genotoxicity potential of vinegar-processed Genkwa Flos. Regul Toxicol Pharmacol. 2015:72:386-93.

24. Enbom ET, Le MD, Oesterich L, Rutgers J, French SW. Mechanism of hepatotoxicity due to black cohosh (Cimicifuga racemosa): histological, immunohistochemical and electron microscopy analysis of two liver biopsies with clinical correlation. Exp Mol Pathol. 2014;96:279-83.

25. Muqeet Adnan M, Khan M, Hashmi S, Hamza M, AbdulMujeeb S, Amer S. Black cohosh and liver toxicity: is there a relationship? Case Rep Gastrointest Med. 2014;2014:860614.

26. KFDA. Good laboratory practice regulation for non-clinical laboratory studies (notification no. 2012-61). 2014. http://nifds.go.kr/cyber/GLP/GLP2014/index. html. Accessed Oct 2014.

27. OECD. 1998. OECD guideline for testing of chemicals, Test No.408: repeated dose 90-day oral toxicity study in rodents.

28. Srivastava KC. Aqueous extracts of onion, garlic and ginger inhibit platelet aggregation and alter arachidonic acid metabolism. Biomed Biochim Acta. 1984;43:S335-46.

29. NRC. Guide for the care and use of laboratory animals. 8th ed. Washington: National Academies Press; 2010.

30. Mudium R, Kolasani B. Anticonvulsant effect of hydroalcoholic seed extract of croton tiglium in rats and mice. J Clin Diagn Res. 2014;8:24-6.

31. Pal PK, Nandi MK, Singh NK. Detoxification of croton tiglium L. seeds by Ayurvedic process of Sodhana. Anc Sci Life. 2014:33:157-61.

32. Madrigales-Ahuatzi D, Perez-Gutierrez RM. Evaluation of anti-inflammatory activity of seeds of Phalaris canariensis. Drug Res. 2016;66:23-7.

33. Cottle W. The treatment of ringworm. Lancet. 1880;115:482-3.

34. Liveing R. The treatment of ringworm by croton oil. Br Med J. 1881;1:227-8.

35. Wang $X$, Zhang FM, Liu ZX, Feng HZ, Yu ZB, Lu YY, Zhai HH, Bai FH, Shi YQ, Lan M, Jin JP, Fan DM. Effects of essential oil from Croton tiglium L. on intestinal transit in mice. J Ethnopharmacol. 2008;117:102-7.

36. Hu J, Gao WY, Gao Y, Ling NS, Huang LQ, Liu CX. M3 muscarinic receptorand $\mathrm{Ca}^{2+}$ influx-mediated muscle contractions induced by croton oil in isolated rabbit jejunum. J Ethnopharmacol. 2010;129:377-80.

37. Wu XA, Zhao YM, Yu NJ. A novel analgesic pyrazine derivative from the leaves of Croton tiglium L. J Asian Nat Prod Res. 2007;9:437-41.

38. Kim JY, Yun JW, Kim YS, Kwon E, Choi HJ, Yeom SC, Kang BC. Mutagenicity and tumor-promoting effects of Tiglium seed extract via PKC and MAPK signaling pathways. Biosci Biotechnol Biochem. 2015;79:374-83.

39. Fetterman BA, Kim BS, Margolin BH, Schildcrout JS, Smith MG, Wagner SM, Zeiger E. Predicting rodent carcinogenicity from mutagenic potency measured in the Ames Salmonella assay. Environ Mol Mutagen. 1997;29: 312-22.

40. Bolognesi C. Genotoxicity of pesticides: a review of human biomonitoring studies. Mutat Res. 2003:543:251-72.

41. Kang $\mathrm{HG}$, Jeong $\mathrm{SH}, \mathrm{Cho} \mathrm{JH}$. Antimutagenic and anticarcinogenic effect of methanol extracts of Petasites japonicus maxim leaves. J Vet Sci. 2010;11:51-8

42. Liu Y, Zhang X, Li ZJ, Chen XH. Up-regulation of CX43 expression and GJIC function in acute leukemia bone marrow stromal cells post-chemotherapy. Leuk Res. 2010;34:631-40.

43. Tanaka R, Tokuda H, Ezaki Y. Cancer chemopreventive activity of "rosin" constituents of Pinus spez. and their derivatives in two-stage mouse skin carcinogenesis test. Phytomedicine. 2008;15:985-92.

44. Lim KT, Lim V, Chin JH. Subacute oral toxicity study of ethanolic leaves extracts of Strobilanthes crispus in rats. Asian Pac J Trop Biomed. 2012;2: $948-52$.

45. Worasuttayangkurn L, Watcharasit P, Rangkadilok N, Suntararuks S, Khamkong P, Satayavivad J. Safety evaluation of longan seed extract: acute and repeated oral administration. Food Chem Toxicol. 2012;50:3949-55.

46. Vaidya VS, Ozer JS, Dieterle F, Collings FB, Ramirez V, Troth S, Muniappa N, Thudium D, Gerhold D, Holder DJ, Bobadilla NA, Marrer E, Perentes E, Cordier A, Vonderscher J, Maurer G, Goering PL, Sistare FD, Bonventre JV. Kidney injury molecule-1 outperforms traditional biomarkers of kidney injury in preclinical biomarker qualification studies. Nat Biotechnol. 2010;28:478-85.

47. Chen Y, Brott D, Luo W, Gangl E, Kamendi H, Barthlow H, Lengel D, Fikes J, Kinter L, Valentin JP, Bialecki R. Assessment of cisplatin-induced kidney injury using an integrated rodent platform. Toxicol Appl Pharmacol. 2013; 268:352-61.

48. Mroueh M, Saab Y, Rizkallah R. Hepatoprotective activity of Centaurium erythraea on acetaminophen-induced hepatotoxicity in rats. Phytother Res. 2004;18:431-3. 
49. Ozdil B, Kece C, Cosar A, Akkiz H, Sandikci M. Potential benefits of combined $\mathrm{N}$-acetylcysteine and ciprofloxacin therapy in partial biliary obstruction. J Clin Pharmacol. 2010;50:1414-9.

50. Mukinda JT, Syce JA. Acute and chronic toxicity of the aqueous extract of Artemisia afra in rodents. J Ethnopharmacol. 2007;112:138-44.

51. Han YD, Song SY, Lee JH, Lee DS, Yoon HC. Multienzyme-modified biosensing surface for the electrochemical analysis of aspartate transaminase and alanine transaminase in human plasma. Anal Bioanal Chem. 2011:400:797-805.

52. Tomizawa M, Kawanabe Y, Shinozaki F, Sato S, Motoyoshi Y, Sugiyama T, Yamamoto S, Sueishi M. Triglyceride is strongly associated with nonalcoholic fatty liver disease among markers of hyperlipidemia and diabetes. Biomed Rep. 2014;2:633-6.

53. Reagan-Shaw S, Nihal M, Ahmad N. Dose translation from animal to human studies revisited. FASEB J. 2008:22:659-61.

Ready to submit your research? Choose BMC and benefit from:

- fast, convenient online submission

- thorough peer review by experienced researchers in your field

- rapid publication on acceptance

- support for research data, including large and complex data types

- gold Open Access which fosters wider collaboration and increased citations

- maximum visibility for your research: over $100 \mathrm{M}$ website views per year

At $\mathrm{BMC}$, research is always in progress.

Learn more biomedcentral.com/submissions 